$\zeta<\alpha$, toute droite située dans $P$ et distincte de chaque droite $d_{\xi}$, où $\xi \leqslant \zeta$, contienne au plus deux points de la suite $\left\{q_{\xi}\right\}_{\xi \leqslant 5}$. Il en résulte immédiatement que toute droite située dans $P$ et distincte de chaque droite $d_{\xi}$, où $\xi<\alpha$, contient au plus deux points de la suite $\left\{q_{\xi}\right\}_{\xi<\alpha}$.

Soit $T_{\alpha}$ l'ensemble formé de toutes les droites $d_{\xi}$, où $\xi<\alpha$, et de toutes les droites du plan $P$ qui passent par deux points au moins de la suite $\left\{q_{\xi}\right\}_{\xi<<}$. Comme $a<\varphi$, l'ensemble $T_{\alpha}$ est de puissance $<2^{N_{0}}$. Les la suite $\left\{q_{\xi}\right\}<a$. Comm de la droite $d_{\alpha}$ arec les droites de $T_{\alpha}$, distinctes points d'intersection $d_{\alpha}$, forment donc un ensemble $E_{a}$ de puissance $<2^{* .}$ et la droite $d_{c}$ contient $2^{N_{0}}$ points qui n'appartiennent ni à $E_{\alpha}$ ni à la suite $\left\{q_{\xi}\right\}_{\xi<\alpha}$. Si l'ensemble des points de $d_{r c}$ qui sont des termes de la suite $\left\{q_{\xi}\right\}_{\xi<\alpha}$ est de puissance $\mathrm{m}_{d_{\alpha}}$, posons $q_{a}=q_{1}$; dans le cas contraire, soit $q_{*}$ le premier terme de la suite $\left\{p_{\xi}\right\}_{\xi<\varphi}$, tel que $q_{\alpha} \bar{\epsilon} E_{\alpha}$ et $q_{\alpha} \neq q_{\xi}$ pour $\xi<\alpha$.

On voit sans peine que toute droite $d$ située dans le plan et distincte de chaque droite $d_{\xi}$, où $\xi \leqslant \alpha$, contient au plus deux points de la suite $\left\{q_{\xi}\right\} \leqslant \because$.

La suite transfinie $\left\{q_{\xi}\right\}_{5<\varphi}$ est ainsi définie par l'induction transfinie. Démontrer que l'ensemble $S$ de tous les termes de cette suite satisfait aux conditions du théorème n'offre pas de difficulté.

\section{An Extension of Sperner's Lemma, with Applications to Closed-Set Coverings and Fixed Points}

By

\section{F. Bage mihl (Rochester, N. Y.)}

I. Introduction. The methods used in this paper are elosely patterned after, and intended to enlarge to some extent the range of, those developed by Sperner, Knaster, Kuratowski, and Mazurkiewicz in [4] and [2]. We first introduce below the notion of an n-dimensional m-plex, which is, roughly speaking, what one obtains from an $n$-dimensional simplex by cutting out a finite number, $m-1$, of $n$-dimensional simplexes. Sperner's Lemma (see [2]; [3], p. 193; [4]) is then sharpened and extended (Lemma 1, Corollary 1) to $m$-plexes satisfying certain simple conditions (pertaining either to the nature of $m$ or to the orientation of the constituent simplexes). This extension is applied to obtain generalizations (Theorem 1, Corollary 2) of theorems - one of Knaster, Kuratowski, and Mazurkiewicz (see [2]; [3], p. 194), which they used to give a proof of Brouwer's Fixed-Point Theorem, and one of Sperner (see [2]; [3], p. 194; [4]), which he used to give a proof of the invariance of dimension - on closed-set coverings; a fixedpoint theorem (Theorem 2) for $n$-dimensional $m$-plexes with $m$ odd, derived along the lines of the proof of Brouwer's Theorem given in [2] (or [3], p. 196); a corollary (Corollary 3) on retraction; and a generalization (Theorem 3) of Kakutani's theorem [1] on fixed points.

II. Preliminaries. Let $S$ be an $n$-dimensional (closed) simplex with vertices $v_{0}, v_{1}, \ldots, v_{n}$; we shall write $S=\left(v_{0} v_{1} \ldots v_{n}\right)$. Its $k$-dimensional $(0 \leqslant k \leqslant n)$ face with vertices $v_{i_{0}}, v_{i_{1}}, \ldots, v_{i_{k}}$ will be denoted by $v_{i_{0}} v_{i_{1}} \ldots v_{i_{k}}$. If $n>0$, we shall denote by $S^{+}=+\left(v_{0} v_{1} \ldots v_{n}\right)$ the oriented simplex obtained from $S$ by giving its vertices the order of succession indicated by the order in which these vertices are written down.

An oriented $n$-dimensional simplex $+\left(v_{0}^{\prime} v_{1}^{\prime} \ldots v_{n}^{\prime}\right)$ is said to have the same orientation as $+\left(v_{0} v_{1} \ldots v_{n}\right)$, if, and only if, there exists a continuous deformation $D\left\{+\left(v_{0}^{\prime} v_{1}^{\prime} \ldots v_{n}^{\prime}\right)\right\}=+\left(v_{0} v_{1} \ldots v_{n}\right)$ such that $D\left(v_{i}^{\prime}\right)=v_{i}(0 \leqslant i \leqslant n)$. If $+\left(v_{0}^{\prime} v_{1}^{\prime} \ldots v_{n}^{\prime}\right)$ does not have the same orientation as $+\left(v_{0} v_{1} \ldots v_{n}\right)$, then it is said to have the opposite orientation. 
Suppose that $n \geqslant 1$. Let $S_{1}=\left(v_{0}^{(1)} v_{1}^{(1)} \ldots v_{n}^{(1)}\right), S_{2}=\left(v_{0}^{(2)} v_{1}^{(2)} \ldots v_{n}^{(2)}\right)$, $\ldots, S_{m}=\left(v_{0}^{(m)} v_{1}^{(m)} \ldots v_{n}^{(m)}\right) \quad(m \geqslant 1)$ be $n$-dimensional simplexes satisfying the following conditions:

(I) $\quad S_{i} C S_{1} \quad(i>1)$

(II) the frontier of $S_{1}$ contains no point of $S_{i} \quad(i>1)$;

(III) $S_{i} \cap S_{j}=0 \quad(i, j>1 ; i \neq j)$

Denote by $S_{1}\left[S_{2} S_{3} \ldots S_{m}\right]$ the (closed) set of points of $S_{1}$ obtained by deleting from $S_{1}$ all points of $S_{i}(i>1)$ which do not belong to the frontier of $S_{i}$. We. call $S_{1}\left[S_{2} S_{3} \ldots S_{m}\right]$ an $n$-dimensional m-plex $(m=1$, $S_{1}=$ simplex; $m=2, S_{1}\left[S_{2}\right]=$ duplex; $m=3, S_{1}\left[S_{2} S_{3}\right]=$ triplex; etc.). If each $S_{i}$ is given one of the two possible orientations to form an oriented simplex $S_{i}^{+}$, then we speak of an oriented m-plex $S_{1}^{+}\left[S_{2}^{+} S_{3}^{+} \ldots S_{m}^{+}\right]$. The vertices of $S_{1}, S_{2}, \ldots, S_{m}$ are called the vertices of the $m$-plex, and the $k$-dimensional $(0 \leqslant k<n)$ faces of $S_{1}, S_{2}, \ldots, S_{m}$ are called the $k$-dimensional faces of the m-plex. It is convenient to regard the $m$-plex itself as its $n$-dimensional face, and to denote this face by $r_{0}^{(1)} l_{1}^{(1)} \ldots v_{n}^{(1)}$.

We say that an $n$-dimensional $m$-plex is divided simplicially into subsimplexes, if it is divided into a finite number of $n$-dimensional simplexes, the intersection of every pair of which is either the empty set or a common $k$-dimensional face.

Let an $n$-dimensional $m$-plex $S_{1}\left[S_{2} S_{3} \ldots S_{m}\right]$ (oriented or not) be divided simplicially into subsimplexes. If, with every vertex $w$ of these subsimplexes, there is associated a number $\varphi(w)$ such that

(1) if $w$ lies on a k-dimensional side $v_{i_{0}}^{(j)} v_{i_{1}}^{(j)} \ldots v_{i_{k}}^{(j)}$ of the

$m$-plex, then $\varphi(w)$ is one of the numbers $i_{0}, i_{1}, \ldots, i_{k}$,

then $\varphi(w)$ will be called a vertex function of this simplicial division of the $m$-plex.

Relative to a particular vertex function $\varphi$ of a specific simplicial division of a given $m$-plex, a subsimplex $\left(w_{0} w_{1} \ldots w_{n}\right)$ such that $\varphi\left(u_{i}\right)=i$ $(0 \leqslant i \leqslant n)$ will be called a representative subsimplex. Let $\varrho$ stand for the number of representative subsimplexes.

Suppose that $\varphi(w)$ is a vertex function of a simplicial division of an $n$-dimensional oriented $m$-plex $S_{1}^{+}\left[S_{2}^{+} S_{3}^{+} \ldots S_{m}^{+}\right]$, where $S_{1}^{+}=+\left(v_{0}^{(1)} v_{1}^{(1)} \ldots v_{n}^{(1)}\right)$, and that $+\left(w_{0} w_{1} \ldots w_{n}\right)$ is an oriented representative subsimplex $\left(\varphi\left(x_{i}\right)=i\right.$ for $0 \leqslant i \leqslant n)$. If $+\left(w_{0} w_{1} \ldots w_{n}\right)$ has the same orientation as $+\left(v_{0}^{(1)} v_{1}^{(1)} \ldots v_{n}^{(1)}\right)$, we call the former a positive representative subsimplex, otherwise, a negative representative subsimplex. Denote the number of positive (negative) representative subsimplexes by $\varrho_{P}\left(\varrho_{N}\right)$. Obviously $\varrho_{2} \varrho_{P}+\varrho_{N}$.

Let the number of the simplexes $s_{2}^{+}, S_{3}^{+}, \ldots, S_{m}^{+}$whose orientation is the same as (opposite of) that of $S_{1}^{+}$be $\pi(\nu)$, so that we have $\pi+\nu=m-1$; such a simplex will be referred to as a $\pi^{-}\left(y_{-}\right)$simplex.

Consider a subsimplex $T=\left(t_{0} t_{1} \ldots t_{n}\right)$ of a simplicial division, such that $\varphi\left(t_{i}\right)=i(0 \leqslant i \leqslant n-1)$. If the orientation of $+\left(t_{0} t_{1} \ldots t_{n}\right)$ is the same as (opposite of) that of $+\left(v_{0}^{(1)} v_{1}^{(1)} \ldots v_{n}^{(1)}\right)$, we say that $+t_{0} t_{1} \ldots t_{n-1}$ is a positive (negative) representative face of $T$. Let us denote the number of positive (negative) representative faces of the subsimplex $T$ by $\alpha_{P}(T)\left(\alpha_{N}(T)\right)$. When we refer to $+t_{0}^{\prime} t_{1}^{\prime} \ldots t_{n-1}^{\prime}$ as a positive (negative) $r e-$ presentative face, we mean simply that it is a positive (negative) representative face of some subsimplex $T^{\prime}$. Because of the assumed simpliciality of the division, if $+t_{0}^{\prime \prime} t_{1}^{\prime \prime} \ldots t_{n-1}^{\prime \prime}$ is an $(n-1)$-dimensional face of some subsimplex, this face is on the frontier of $S_{1}^{+}\left[S_{2}^{+} S_{3}^{+} \ldots S_{m}^{+}\right]$if, and only if, it is a face of precisely one subsimplex. On the other hand, it is not on the frontier of $S_{1}^{+}\left[S_{2}^{+} S_{3}^{+} \ldots S_{m}^{+}\right]$if, and only if, it is a face of precisely two subsimplexes. In this case, if $\varphi\left(t_{i}^{\prime \prime}\right)=i(0 \leqslant i \leqslant n-1)$, it is a positive representative face of one of these subsimplexes, and a negative representative face of the other one. Let the number of positive (negative) representative faces on the frontier of $S_{1}^{+}\left[S_{2}^{+} S_{3}^{+} \ldots S_{m}^{+}\right]$be $\sigma_{P_{1}}\left(\sigma_{N}\right)$, and let the number of positive (negative) representative faces on the frontier of $S_{j}^{+}(1 \leqslant j \leqslant m)$ be $\sigma_{P}^{(j)}\left(\sigma_{N}^{(j)}\right)$.

By the term representative face we shall mean an $(n-1)$-dimensional face $w_{0} w_{1} \ldots w_{n-1}$ of some subsimplex of the division, such that $\varphi\left(w_{i}\right)=i$ $(0 \leqslant i \leqslant n-1)$. Let $\psi$ stand for the number of representative faces which are not on the frontier of $S_{1}^{+}\left[S_{2}^{+} S_{3}^{+} \ldots S_{m}^{+}\right]$.

If $w_{0} w_{1} \ldots w_{n-1}$ is a representative face on the frontier of $S_{1}^{+}\left[S_{2}^{+} S_{3}^{+} \ldots S_{m}^{+}\right]$, then, because of (1), $w_{0} w_{1} \ldots v_{n-1}$ must lie on $v_{0}^{(j)} v_{1}^{(j)} \ldots v_{n-1}^{(j)}$ for some $j$. If, in addition, $j>1$ and the orientation of $+w_{0} w_{1} \ldots v_{n-1}$ is the same as (opposite of) that of $+v_{0}^{(i)} v_{1}^{(j)} \ldots v_{n-1}^{(j)}$, then $+w_{0} w_{1} \ldots w_{n-1}$ is a positive (negative) representative face if $S_{j}^{+}$is a $v$-simplex, but a negative (positive) representative face if $S_{j}^{+}$is a $\pi$-simplex.

By an incomplete subsimplex (let the number of such subsimplexes be $x)$ we mean a subsimplex $T=\left(t_{0} t_{1} \ldots t_{n}\right)$ such that $\varphi\left(t_{i}\right)=i(0 \leqslant i \leqslant n-1)$ and $\varphi\left(t_{n}\right) \neq n$. Clearly $\varphi\left(t_{n}\right)=i_{0}$, where $0 \leqslant i_{0} \leqslant n-1$. If $+t_{0} t_{1} \ldots t_{i_{0}} \ldots t_{n-1}$ is a positive (negative) representative face of $T$, then $+t_{0} t_{1} \ldots t_{n} \ldots t_{n-1}$ is a negative (positive) representative face of $T$, and these two are the only representative faces of $T$.

111. Lemma 1. Let $\varphi$ be a vertex function of a simplicial division of an oriented $n$-dimensional m-plex. Then

$$
\varrho_{P}+\pi=\varrho_{N}+\nu+1 .
$$

Proof. Let the $m$-plex in question be $S_{1}^{+}\left[S_{2}^{+} S_{3}^{+} \ldots S_{m}^{+}\right]$. We shall prove the lemma by induction on $n$, verifying it for $n=1$, and, simultaneously, for $n$ under the assumption that it is true for some $n-1 \geqslant 1$. We have
(3)
$\Sigma \alpha_{P}(T)=\varrho_{P}+\chi$
and
(3') $\Sigma \alpha_{N}(T)=\varrho_{N}+\chi$.

Here, as well as in what follows, the summation is extended over all subsimplexes $T$ of the simplicial division. 
We shall prove (3); the proof of $\left(3^{\prime}\right)$ is entirely analogous. Given any $T$, it is one of the following (the numbers indicate the contribution of $T$ to $\Sigma \alpha_{P}(T), \varrho_{P}, \chi$, respectively):

(a) a positive representative subsimplex $(1=1+0)$,

(b) an incomplete subsimplex $(1=0+1)$,

(c) neither (a) nor (b) $(0=0+0)$.

Each $T$ thus contributes the same to the left as to the right of (3), so that the equality holds.

We also have

$$
\Sigma a_{P}(T)=\sigma_{P}+\psi \quad \text { and } \quad\left(4^{\prime}\right) \quad \Sigma a_{N}(T)=\sigma_{N}+\psi
$$

We may again confine ourselves to the first equality. Consider any $(n-1)$-dimensional face of any subsimplex of the simplicial division. This face is one of the following (the numbers referring this time to the terms in (4)):

(a') a positive representative face on the frontier of the m-plex $(1=1+0)$ $(0=0+0)$

$\left(b^{\prime}\right)$ a negative representative face on the frontier of the $m$-plex

$\left(c^{\prime}\right)$ a representative face not on the frontier of the $m$-plex $(1=0+1)$,

$\left(d^{\prime}\right)$ not a representative face $(0=0+0)$.

Evidently (4) is true.

From (3) and (4) we obtain

and $\left(3^{\prime}\right)$ and $\left(4^{\prime}\right)$ yield

$$
\varrho_{P}=\sigma_{P}+(\psi-\gamma),
$$

$$
\varrho_{N}=\sigma_{N}+(\psi-\chi) .
$$

Eliminating $\psi-\chi$ from $(5)$ and $\left(5^{\prime}\right)$, we find that

$$
\varrho_{P}-\varrho_{N}=\sigma_{P}-\sigma_{N}=\sum_{j=1}^{m}\left(\sigma_{P}^{(j)}-\sigma_{N}^{(j)}\right) .
$$

If $n=1$, it is easily seen that $\sigma_{P}^{(1)}=1, \sigma_{N}^{(1)}=0$, and that $\sigma_{N}^{(1)}=1$ or 0 , and $\sigma_{P}^{(j)}=0$ or 1 , according as $S_{j}^{+}(j>1)$ is a $\pi$ - or a $y$-simplex. Hence, $\sigma_{P}-\sigma_{N}=1-\pi+v$, and substituting this value in (6), we see that (2) is true for $n=1$.

If $n>1$, the induction hypothesis, applied to the vertex function $\varphi$ of the simplicial division of $+v_{0}^{(1)} v_{1}^{(1)} \ldots v_{n-1}^{(1)}$ induced by the given simplicial division of the $m$-plex, yields $\sigma_{P}^{(1)}-\sigma_{N}^{(1)}=1$. The same argument applied to $+v_{0}^{(j)} v_{1}^{(j)} \ldots v_{n-1}^{(j)}(j>1)$ shows that $\sigma_{P}^{(j)}-\sigma_{N}^{(j)}=-1$ or +1 according as $S_{j}^{+}$is a $\pi$ - or a $\nu$-simplex. Thus again $\sigma_{p}-\sigma_{N}=1-\pi+\nu$.

This completes the proof of the lemma.
Corollury 1. If $\pi \neq \nu+1$, then $\varrho>0$. If $m$ is odd, then (even if the $m$-plex is not oriented) $\varrho$ is odd (and hence $\varrho>0$ ).

For if we add $\varrho_{N}$ to both sides of (2), we obtain

$$
\varrho=2 \varrho_{N}+v-\pi+1 ;
$$

which shows that $\varrho>0$ if $\nu \geqslant \pi$. The addition of $\varrho_{P}$ to both sides of (2) yields

$$
\varrho=2 \varrho_{P}+\pi-\nu-1,
$$

which implies that $\varrho>0$ if $\pi>v+1$. If we combine these two results, we get the first part of Corollary 1, and the second part follows easily from the fact that $m=\pi+\nu+1$.

The assertion that $\varrho$ is odd if $m=1$, is "Sperner's Lemma".

IV. Theorem 1. Let $C_{0}, C_{1}, \ldots, C_{n}$ be closed sets such that every $k$-dimensional face $v_{i_{0}}^{(j)} v_{i_{1}}^{(j)} \ldots v_{i_{k}}^{(j)}$ of the n-dimensional m-plex $S_{1}^{+}\left[S_{2}^{+} S_{3}^{+} \ldots S_{m}^{+}\right]$ is contained in the union $C_{i_{0}} \cup C_{i_{1}} \cup \ldots \cup C_{i_{k}}$. If $\pi \neq \nu+1$, or if $m$ is odd (in which case it is not necessary to assume that the m-plex is oriented), then $C_{0} \cap C_{1} \cap \ldots \cap C_{n} \neq 0$.

Proof. For a fixed natural number $d$, consider a simplicial division of the given $m$-plex into subsimplexes of diameter less than $1 / d$. Let $w$ be an arbitrary vertex of any one of these subsimplexes, and let $v_{i_{0}}^{(j)} v_{i_{1}}^{(j)} \ldots v_{i_{k}}^{(j)}$ be that face (of the m-plex) of lowest dimension, which contains $w$. By hypothesis, the face $v_{i_{0}}^{(j)} v_{i_{1}}^{(j)} \ldots v_{i_{k}}^{(j)}$ is contained in the union $C_{i_{0}} \cup C_{i_{1}} \cup \ldots \cup C_{i_{k}}$, and consequently there is at least one (specific) $i_{h}(0 \leqslant h \leqslant k)$ such that Put

$$
w \in C_{i_{h}} .
$$

$$
\varphi(w)=i_{h} .
$$

Then (1) is satisfied, so that $\varphi$ is a vertex function of the simplicial division of the $m$-plex. From (7) and (8) we see that

$$
w \in C_{q(w)} \text {. }
$$

By hypothesis, either $\pi \neq v+1$ or $m$ is odd, so that according to Corollary 1 , there exists a representative subsimplex, which we shall denote by $\left(w_{0}^{d} w_{1}^{d} \ldots w_{n}^{d}\right)$, where $\varphi\left(w_{i}^{d}\right)=i(0 \leqslant i \leqslant n)$. From (9) we obtain

$$
w_{i}^{d} \in C_{i}
$$

Now let $d$ tend to infinity. We may assume that $y=\lim _{d \rightarrow \infty} w_{0}^{d}$ exists, and since the diameters, $1 / d$, of the subsimplexes tend to 0 , we have

$$
y=\lim _{d \rightarrow \infty} x_{i}^{d}
$$

$(0 \leqslant i \leqslant n)$.

Since, by assumption, the sets $C_{i}$ are closed, it follows from (10) and (11) that $y \in C_{0} \cap C_{1} \cap \ldots \cap C_{n}$, and the theorem is proved. 
Corollary 2. Let the n-dimensional m-plex $S_{1}^{+}\left[\mathcal{S}_{2}^{+} S_{3}^{+} \ldots S_{m}^{+}\right]$be contained in the union of the closed sets $C_{0}, C_{1}, \ldots, C_{n}$, and let the intersection of the face $s_{i}^{(j)}=v_{0}^{(j)} v_{1}^{(j)} \ldots v_{i-1}^{(j)} v_{i+1}^{(j)} \ldots v_{n}^{(j)}$ with $C_{i}(0 \leqslant i \leqslant n ; 1 \leqslant j \leqslant m)$ be empty. If $\pi \neq v+1$, or if $m$ is odd (in which case it is not necessary to assume that the m-plex is oriented), then $C_{0} \cap C_{1} \cap \ldots \cap C_{n} \neq 0$.

Proof. An arbitrary face $v_{i_{0}}^{(j)} v_{i_{1}}^{(j)} \ldots v_{i_{k}}^{(j)}$ of the $m$-plex is contained in any face $s_{i}^{(j)}$ (with the same $j$ ) for which $i$ is none of the numbers $i_{0}, i_{1}, \ldots, i_{k}$; and therefore, since, by the hypothesis of Corollary $2, v_{i_{0}}^{(j)} v_{i_{1}}^{(j)} \ldots v_{i_{k}}^{(j)}$ contains no point of any $C_{i}$ with such an index $i$, this face must be contained in the union of the remaining closer sets. Thus, the hypothesis of Theorem 1 is satisfied, which implies the conclusion of Corollary 2.

V. Theorrm 2. Let $S_{1}\left[S_{2} S_{3} \ldots S_{m}\right]$ be an n-dimensional m-plex, with $m$ odd, and let $f$ be a continuous mapping of this m-plex into the n-dimensional Euclidean space containing it, such that the frontier of the simplex $S_{j}$ $(1 \leqslant j \leqslant m)$ is mapped into the simplex $S_{j}$. Then the m-plex has at least one fixed point under the mapping $f$.

Proof. Let $S_{j}=\left(v_{0}^{(j)} v_{1}^{(j)} \ldots v_{n}^{(j)}\right) \quad(1 \leqslant j \leqslant m)$.

Assume first that every $k$-dimensional face $v_{i_{0}}^{(j)} v_{i_{1}}^{(j)} \ldots v_{i_{k}}^{(j)}$ of $S_{j} \quad(j>1)$ is parallel to the corresponding face $v_{i_{0}}^{(1)} v_{i_{1}}^{(1)} \ldots v_{i_{k}}^{(1)}$ of $S_{1}$. Regard the vertices $v_{i}^{(\mathbf{1})}(0 \leqslant i \leqslant n)$ as vectors of $n$-dimensional space, and let the points $x$ of the given $m$-plex be represented barycentrically in the form

$$
x=b_{0} v_{0}^{(1)}+b_{1} v_{1}^{(1)}+\ldots+b_{n} v_{n}^{(1)}
$$

where every $b_{i} \geqslant 0$, and $b_{0}+b_{1}+\ldots+b_{n}=1$. Let $x^{\prime}=f(x)$. Then $x^{\prime}$ has a unique barycentric representation:

$$
x^{\prime}=b_{0}^{\prime} v_{0}^{(1)}+b_{1}^{\prime} v_{1}^{(1)}+\ldots+b_{n}^{\prime} v_{n}^{(1)} .
$$

Because of the assumed special position of the simplexes $s_{j}$ relative to $S_{1}$, there exist fixed, nonnegative numbers $a_{i}^{(j)}(0 \leqslant i \leqslant n ; 1 \leqslant j \leqslant m)$ with the following property: If $x$ is on the $(n-1)$-dimensional face $v_{0}^{(j)} v_{1}^{(j)} \ldots v_{h-1}^{(j)} v_{h+1}^{(j)} \ldots v_{n}^{(j)}$ of $S_{j}$, then, for the $h$-th coordinate of $x$ in (12), we have

$$
b_{h}=a_{h}^{(j)}
$$

(In particular, if $j=1, a_{i}^{(j)}=0$ for every $i$ ). Since, by hypothesis, the frontier of $S_{j}$ is mapped into $S_{j}$,

$$
b_{h}^{\prime} \geqslant a_{h}^{(j)} \text {. }
$$

Consequently, if $x$ is on the $k$-dimensional face $v_{i_{0}}^{(j)} v_{i_{1}}^{(j)} \ldots v_{i k}^{(j)}$ of $S_{j}$, and if $v_{i_{1}^{\prime}}^{(j)}, v_{i_{2}^{\prime}}^{(j)}, \ldots, v_{i_{n-k}^{(j)}}^{(j)}$ are the remaining vertices of $S_{j}$, then

$$
\begin{gathered}
b_{i_{1}^{\prime}}=a_{i_{1}^{\prime}}^{(j)}, b_{i_{2}^{\prime}}=a_{i_{2}^{\prime}}^{(j)}, \ldots, b_{i_{n-k}^{\prime}}=a_{i_{n-k}^{\prime}}^{(j)}, \\
b_{i_{0}}+b_{i_{1}}+\ldots+b_{i_{k}}=1-\left(a_{i_{1}^{\prime}}^{(j)}+a_{i_{2}^{\prime}}^{(j)}+\ldots+a_{i_{n-k}^{\prime}}^{(j)}\right),
\end{gathered}
$$

and

$$
b_{i_{1}^{\prime}}^{\prime} \geqslant a_{i_{1}^{\prime}}^{(j)}, \quad b_{i_{2}}^{\prime} \geqslant a_{i_{2}^{\prime}}^{(j)}, \ldots, b_{i_{n-k}^{\prime}}^{\prime} \geqslant a_{i_{n-k}^{\prime}}^{(j)} .
$$

Let $C_{i}$ be the set of points $x$ of the $m$-plex, for which $b_{i}^{\prime} \leqslant b_{i}$. Due to the continuity of $f$, the sets $C_{0}, C_{1}, \ldots, C_{n}$ are closer.

We shall show that they satisfy the hypothesis of Theorem 1. Indeed, suppose that there were a point $x$ of a $k$-dimensional face $v_{i_{0}}^{(j)} v_{i_{1}}^{(j)} \ldots v_{i_{k}}^{(j)}$ of the $m$-plex, which did not belong to the union $C_{i_{0}} \cup C_{i_{1}} \cup \ldots \cup C_{i_{k}}$. Then we should have

and hence, by (13),

$$
b_{i_{0}}^{\prime}>b_{i_{0}}, \quad b_{i_{1}}^{\prime}>b_{i_{1}}, \ldots, b_{i_{k}}^{\prime}>b_{i_{k}},
$$

$$
b_{i_{0}}^{\prime}+b_{i_{1}}^{\prime}+\ldots+b_{i_{k}}^{\prime}>1-\left(a_{i_{1}^{\prime}}^{(j)}+a_{i_{2}^{\prime}}^{(j)}+\ldots+a_{i_{n-k}^{\prime}}^{(j)}\right) .
$$

On the other hand, by (14),

$$
b_{i_{1}^{\prime}}^{\prime}+b_{i_{2}^{\prime}}^{\prime}+\ldots+b_{i_{n-k}^{\prime}}^{\prime} \geqslant a_{i_{\perp}^{\prime}}^{(j)}+a_{i_{2}^{\prime}}^{(j)}+\ldots+a_{i_{n-k}^{\prime}}^{(j)} .
$$

Combining (15) and (16), we should obtain

which is impossible.

$$
b_{0}^{\prime}+b_{1}^{\prime}+\ldots+b_{n}^{\prime}>1
$$

Let $y \in C_{0} \cap C_{1} \cap \ldots \cap C_{n} ; y$ exists according to Theorem 1 . By the definition of $C_{i}$, we have, for $x=y$ :

$$
b_{0}^{\prime} \leqslant b_{0}, \quad b_{1}^{\prime} \leqslant b_{1}, \ldots, b_{n}^{\prime} \leqslant b_{n},
$$

and hence

$$
1=b_{0}^{\prime}+b_{1}^{\prime}+\ldots+b_{n}^{\prime} \leqslant b_{0}+b_{1}+\ldots+b_{n}=1 .
$$

Consequently $b_{i}^{\prime}=b_{i}$, and therefore $y^{\prime}=y, i . e ., y$ is a fixed point under the mapping $f$.

Now let us remove from our m-plex $S_{1}\left[S_{2} S_{3} \ldots S_{m}\right]$ the special assumption made at the beginning of the proof. Let $S_{1}^{*}\left[S_{2}^{*} S_{3}^{*} \ldots S_{m}^{*}\right]$ be an auxiliary $m$-plex, however, for which the special assumption does hold, and which is the image of $S_{1}\left[S_{2} S_{3} \ldots S_{m}\right]$ under a homeomorphism $g$ of $S_{1}$ onto $S_{1}^{*}$ such that $g\left(S_{j}\right)=S_{j}^{*}(1 \leqslant j \leqslant m)$. Then, according to the first part of our proof, the mapping $g f g^{-1}$ has a fixed point $x^{*} \epsilon^{\prime} S_{1}^{*}\left[S_{2}^{*} S_{3}^{*} \ldots S_{m}^{*}\right]$, so that

and hence

$$
g^{-1}\left(x^{*}\right)=x^{*},
$$

$$
f g^{-1}\left(x^{*}\right)=g^{-1}\left(x^{*}\right),
$$

which means that the point $g^{-1}\left(x^{*}\right) \in S_{1}\left[S_{2} S_{3} \ldots S_{m}\right]$ is fixed under $f$.

This completes the proof of Theorem 2. 
VI. Remarks. The following example shows that if $\pi=\nu+1$ or if $m$ is even, then the conclusion of Theorem 1 or of Corollary 1 need not hold.

Let $r$ be a nonnegative integer, $S_{1}^{+}=+\left(v_{0}^{(1)} v_{1}^{(1)} \ldots v_{n}^{(1)}\right)$ be an $n$-dimensional oriented simplex, and $w$ be the barycenter of the $(n-1)$-dimensional face, $v_{1}^{(1)} v_{2}^{(1)} \ldots v_{n}^{(1)}$, of $S_{1}^{+}$. (If $n=1$, then $w=v_{1}^{(1)}$ ). On the open segment $v_{0}^{(1)} w$, choose $2 v+1$ points, $z_{0}, y_{1}, z_{1}, y_{2}, z_{2}, \ldots, y_{\nu}, z_{\nu}$ in the order $v_{0}^{(1)}, z_{0}, y_{1}, z_{1}, y_{2}, z_{2}, \ldots, y_{i}, z_{v}, w$. It will be convenient to denote $v_{0}^{(1)}$ by $y_{0}$.

Call the $n$-dimensional simplex

$$
\left(y_{i} \approx_{i} v_{1}^{(1)} v_{2}^{(1)} \ldots v_{j-1}^{(1)} v_{j+1}^{(1)} \ldots v_{n}^{(1)}\right) \quad(0 \leqslant i \leqslant \nu ; 1 \leqslant j \leqslant n)
$$

$A_{i \tau(j)}$, where $\tau(j)=j+1$ for $j=1,2, \ldots, n-1$, and $\tau(n)=0$. Put

and

$$
\sigma_{k}^{\prime}=\cup_{0 \leqslant i \leqslant v} A_{i k} \quad(0 \leqslant k \leqslant n ; k \neq 1)
$$

$$
C_{1}^{\prime}=S_{1}-\underset{\substack{0 \leqslant k \leqslant n \\ k \neq 1}}{\bigcup} C_{k}^{\prime}
$$

(where $\bar{X}$ denotes the closure of the set $X$ ). The sets $C_{k}^{\prime}(0 \leqslant k \leqslant n)$ are obviously closed, and

$$
\bigcap_{0 \leqslant k \leqslant n} \sigma_{k}^{\prime}=\left\{z_{0}, y_{1}, z_{1}, y_{2}, z_{2}, \ldots, y_{v}, z_{v}\right\} .
$$

On the open segment $z_{\nu} u$, choose a point $u_{\nu 1}$. If $v>0$, then on the open segment $z_{r} y_{r+1}\left(0 \leqslant r \leqslant v^{\prime}-1\right)$ choose two points, $u_{r 1}, u_{r+1,1}^{\prime}$, in the order $z_{r}, u_{r 1}, u_{r+1,1}^{\prime}, y_{r+1}$. The points chosen all belong to $C_{1}^{\prime}$.

On the open segment $y_{0} z_{0}$, take a point $h_{0}$, and let $H_{0}$ be the $(n-1)$ dimensional hyperplane which contains $h_{0}$ and is perpendicular to the segment $y_{0} w$. If $\nu>0$, then on the open segment $y_{r} z_{r} \quad(1 \leqslant r \leqslant v)$ take two points, $h_{r}^{\prime}, h_{r}$, in the order $y_{r}, h_{r}^{\prime}, h_{r}, z_{r}$. Let $H_{r}^{\prime}, H_{r}$ be the $(n-1)$-dimensional hyperplane which contains $h_{r}^{\prime}, h_{r}$, respectively, and is perpendicular to the segment $y_{0} w$.

In the interior of the simplex $A_{0 k}(0 \leqslant k \leqslant n ; k \neq 1)$, select a point $u_{0 k}$ which belongs to $H_{0}$. If $\nu>0$, then in the interior of the simplex $A_{r k}$ $(1 \leqslant r \leqslant \nu ; 0 \leqslant k \leqslant n ; k \neq 1)$, select two points, $u_{r k}^{\prime}, u_{r k}$, which belong to $H_{r}^{\prime}, H_{r}$, respectively.

Consider the following $2 v+1$ oriented $n$-dimensional simplexes:

$$
\begin{array}{ll}
R_{r}^{+}=+\left(u_{r 0} u_{r 1} \ldots u_{r n}\right) & (0 \leqslant r \leqslant \nu), \\
R_{r^{\prime}}^{+}=+\left(u_{r 0}^{\prime} u_{r_{1}}^{\prime} \ldots u_{r n}^{\prime}\right) & (1 \leqslant r \leqslant \nu) ;
\end{array}
$$

(the simplexes (19) are defined only if $\nu>0$ ). These simplexes are mutually exclusive and lie in the interior of $S_{1}^{+}$, so that they may be regarded as constituent simplexes of an oriented $n$-dimensional m-plex

$R_{1}^{+}\left[R_{0}^{+} R_{1}^{+} \ldots R_{\nu}^{+} R_{1^{\prime}}^{+} R_{2^{\prime}}^{+} \ldots R_{v^{\prime}}^{+}\right]$, where $m=2 \nu+2$, an even number. The $\nu+1$ simplexes (18) are $\pi$-simplexes, the $v$ simplexes (19) are $v$-simplexes, and consequently $\pi=\nu+1$.

Let $O_{i}(0 \leqslant i \leqslant n)$ be the intersection of $C_{i}^{\prime}$ with the $m$-plex just defined; obviously $C_{i}$ is closed. These closed sets and our $m$-plex satisfy the condition expressed in the first sentence of Theorem 1. From the construction of the simplexes (18) and (19), it is clear that the $m$-plex does not contain the points $z_{0}, y_{1}, z_{1}, y_{2}, z_{2}, \ldots, y_{\nu}, z_{\nu}$. This means, if we bear (17) in mind, that

$$
C_{0} \cap C_{1} \cap \ldots \cap C_{n}=0 .
$$

Thus, the conclusion of Theorem 1 is false. This implies, in view of the proof of Theorem 1, that $\varrho=0$ for some vertex function of some simplicial division of our $m$-plex, so that the conclusion of Corollary 1 is false too.

It is also possible to give an example which shows that Corollary 2 may fail to hold if $\pi=\nu+1$ or if $m$ is even.

Theorem 2 is obviously false if $m=2$, for there is no fixed point under the mapping of a duplex $S_{1}\left[S_{2}\right]$ into the barycenter of $S_{2}$.

Theorem 2 has the following corollary (cf. [3], p. 197):

Corollain. 3. The frontier of an n-dimensional m-plex $S_{1}\left[S_{2} S_{3} \ldots S_{m}\right]$, with $m$ odd, is not a retract of the m-plex.

Proof. Let $p$ be a point on the frontier of the $m$-plex; $p$, then, is on the frontier of precisely one $S_{j}$. By $\tilde{p}$ we mean the point of intersection of the frontier of $S_{j}$ with the ray emanating from $p$ and passing through the barycenter of $S_{j}$. Clearly $\tilde{p} \neq p$, and $\underset{\tilde{p}}{\approx}=p$.

Now suppose that there existed a retraction, $f$, of the $m$-plex onto its frontier. Then, by definition (see [3], p. 75),

(20) $f(x)=x$ for every $x$ on the frontier of the m-plex.

According to Theorem 2, the function $\widetilde{f(x)}$ has a fixed point, i.e., there exists a point $x_{0}$ (naturally on the frontier of the $m$-plex) such that $\widetilde{f\left(x_{0}\right)}=x_{0}$. Hence, $f\left(x_{0}\right)=\tilde{x}_{0} \neq x_{0}$, contradicting $(20)$.

Theorem 2 can be used to extend Kakutani's generalization of the fixed-point theorem of Brouwer. The proof of this extension is formally so analogous to Kakutani's proof of his Theorem 1 in [1], that, after making a few necessary remarks, we may refer the reader to Kakutani's paper for the details of the proof. With this in mind, our notation and terminology will be chosen as close to Kakutani's as possible.

Let $S=S_{1}\left[S_{2} S_{3} \ldots S_{m}\right]$ be an $r$-dimensional $m$-plex with $m$ odd, and $R$ be a closed, bounded region (in $r$-dimensional Euclidean space) containing $S$. Denote by $\Re(R)$ the family of all nonempty, closed, convex subsets of $R$. 
A point-to-set mapping $x \rightarrow \Phi(x) \in \mathcal{N}(R)$ of $S$ into $\mathcal{R}(R)$ is called upper semi-continuous, if $\lim x_{n}=x_{0}, y_{n} \in \Phi\left(x_{n}\right)$, and $\lim y_{n}=y_{0}$ imply that $y_{0} \in \Phi\left(x_{0}\right)$. Our extension of Kakutani's theorem may be stated as follows:

Theorem 3. Let $x \rightarrow \mathscr{I}(x)$ be an upper semi-continuous point-to-set mapping of an r-dimensional m-plex $S=S_{1}\left[S_{2} S_{3} \ldots S_{m}\right]$, with $m$ odd, into $\boldsymbol{\Omega}(R)$, such that if $x$ is on the frontier of $S_{j}(1 \leqslant j \leqslant m)$, then $\Phi(x)$ is a subset of the simplex $S_{j}$. Then there exists an $x_{0} \in S$ such that $x_{0} \in \Phi\left(x_{0}\right)$.

By the $n$-th barycentric simplicial subdivision of the $m$-plex $S$, we mean the simplicial division of $s$ determin d by the $n$-th barycentric subdivision of erery subsimplex of some fixed simplicial division of $S$. If, now, in Kakutani's proof, we replace the appeal to Brouwer's Theorem by an appeal to our Theorem 2 proved ahove, and make several obvious minor modifications, we obtain a proof of our Theorem 3 .

\section{Bibliography}

[1] Kakutani, S., A generalization of Brouwer's fixed point theorem, Duke Math. J 8 (1941), p. 457-459.

[2] Knaster, B., Kuratowski, C. and Mazurkiewicz, S., Ein Beweis des Fixpunktsatzes für n-dimensionale Simplexe, Fund. Math. 14 (1929), p. 132-137.

[3] Kuratowski. C., Topologie $1, \Sigma^{\mathrm{d}}$ ed., Warszawa-Wroclaw 1948.

[4] Sperner, E., Neuer Beweis für die Invarianz der Dimensionszahl und des Gebietes, Abh. math. Semin: Hamburg. Univ. 6 (1928), p. 265-272

\section{Sur la caractérisation topologique de l'ensemble des bouts d'une courbe}

Par

\section{B. Knaster et M. Reichbach (Wroclaw)}

Généralités. Nous entendons ici par courbe tout continn (ensemble compact et connexe dans un espace séparable) de dimension 1 au sens de Menger ${ }^{1}$ ), par l'ordre du point $p$ d'un ensemble $E$, en symbole: ord $(p, E)$, le plus petit nombre cardinal pour lequel il existe dans tout entourage de $p$ (ensemble ouvert contenant $p$ ) un entourage $d u$ même point dont la frontière a exactement ce nombre des points communs arec $E$, enfin par bout (extrémité) de $E$ - tout point pour lequel ord $\left.(p, E)=1^{2}\right)$. Cette égalité implique que $p \in E$ lorsque $E$ est fermé.

Le livre - déjà classique - de Menger ${ }^{3}$ ), anquel ces notions sont empruntées, contient des théorèmes dont il résulte en particulier que l'ensemble $C^{1}$ des bouts d'une courbe $C$ quelconque est un $G_{\delta}$ de dimension $\left.\cdot 0^{4}\right)$. La question s'impose, si les deux dernières propriétés nécessaires sont déjà caractéristiques, c'est-à-dire à la fois suffisantes pour qu'un ensemble soit celui des bouts d'une courbe. Les considérations qui suivent donnent réponse à cette question.

Il y a d'abord lieu de fixer ce qu'il y est à caractériser topologiquement. Etant donné un ensemble $B$, l'existence d'une courbe $C$ telle que $B=C^{1}$ n'est un invariant de l'homéomorphie de $B$ ni dans des espaces topologiques fort pauvres (tels, en particulier, que la dendrite $\Delta^{5}$ ) dont nous ferons, à la fin, un usage essentiel pour la solution du problème), ni dans des espaces très vastes (celui de Hilbert par exemple). Envisageons en effet les deux exemples.

1) Voir C. Kuratowski, Topologie I, Monografie Matematyczne. WarszawaWrocław 1948, deuxième édition, p. 162.

2) K. Menger, Kurventheorie, Leipzig-Berlin 1932, p. 97.

) Op. cit., p. 99.

4) Ibidem, p. 105 et 112 .

5) Cf. H. M. Gehman, Concerning the subsets of a plane continuous ourve, Annals of Mathematics 27 (1925-1926), p. 42 et 43 , où une construction analogue est employée à un but différent. La partie de $\Delta$ située au-dessous de l'axe des $x$ est homéomorphe à celle de la courbe de Gehman située au-dessus de cet axe. 